\title{
O manguezal na visão etnoecológica dos pescadores artesanais do Canal de Santa Cruz, Itapissuma, Pernambuco, Brasil
}

\author{
Marcos Antônio Bezerra Carneiro'* \\ Cristiane Maria Rocha Farrapeira ${ }^{2}$ \\ Karla Maria Euzébio da Silva ${ }^{1}$ \\ ${ }^{1}$ Programa de Pós-Graduação em Ensino das Ciências \\ Universidade Federal Rural de Pernambuco (UFRPE) \\ ${ }^{2}$ Departamento Biologia (UFRPE), Rua Dom Manoel de Medeiros, s/n \\ CEP 52-171-900, Recife - PE, Brasil \\ *Autor para correspondência \\ marcosabcarneiro@yahoo.com.br
}

\section{Resumo}

A etnoecologia estuda o modo como as populações humanas interpretam conexões oriundas das interações entre a sociedade e a natureza. A presente pesquisa foi realizada com o objetivo de verificar a relação existente entre os pescadores artesanais de Itapissuma, Pernambuco e o complexo estuário-manguezal. Assim, um grupo de 54 pescadores do Canal de Santa Cruz foi entrevistado, tendo demonstrado um conhecimento acurado sobre a biodiversidade destes ecossistemas, as relações ecológicas que lá se processam e a importância destes recursos naturais para a produção pesqueira. Tais conhecimentos evidenciados refletem uma bagagem cultural onde a arte da pesca é transmitida aos descendentes, através de observações e aprendizagem prática na comunidade tradicional em questão, cuja base econômica familiar advém desses recursos.

Unitermos: complexo estuário-manguezal, etnoecologia, pescadores artesanais, biodiversidade, atividade pesqueira

\section{Abstract}

The mangrove in the ethnoecological perception of artisanal fishermen of the Santa Cruz Channel, Itapissuma, Pernambuco. Ethnoecology studies the way human populations interpret the connections arising from interactions between society and nature. The present research was conducted with the aim of elucidating the relationship between the artisanal fishermen of Itapissuma, Pernambuco, and the estuary-mangrove complex. Thus, a group of 54 fishermen of the Santa Cruz Channel were interviewed. They demonstrated an accurate knowledge about the biodiversity of these ecosystems, the ecological relations operating there, and the importance of these natural resources for fishing production. This knowledge reflects a cultural background in which the art of fishing is transmitted to descendants, through observations and practical learning in the traditional community in question: in such communities, the economic subsistence of families depends on those resources.

Key words: Estuary-mangrove complex, ethnoecology, artisan fishermen, estuarine biodiversity, fishing activity 


\section{Introdução}

Algumas áreas da ciência procuram estudar o conhecimento de comunidades tradicionais sobre o meio ambiente, o que revela um retrato da população e de sua visão do mundo (Albuquerque e Albuquerque, 2005). A etnociência vem se constituindo, no panorama científico, em um diálogo frutífero entre as ciências naturais e as ciências humanas e sociais (Nishida, 2005). Neste campo de saber se destaca a etnobiologia por tratar das percepções, incluindo o estudo dos tipos e usos dos recursos biológicos e a lógica subjacente à sua classificação elaborada por seres humanos em relação ao meio ambiente (Begossi e Figueiredo, 1995).

Segundo Posey (1997), esta ciência estuda essencialmente o conhecimento e as conceituações desenvolvidas por uma sociedade a respeito da biologia, estudando o papel da natureza no sistema de crenças e de adaptação do homem a determinados ambientes. Focando este estudo para o ambiente, emerge uma outra ciência, a etnoecologia, definida como o campo de pesquisa transdisciplinar que estuda o modo como as populações humanas se inserem culturalmente em ecossistemas, através de processos cognitivos e de reações emocionais e comportamentais, no qual se interpretam conexões que emergem das interações entre a sociedade e a natureza. Desta forma, estuda os conhecimentos, crenças, sentimentos e comportamentos que intermedeiam as interações entre as populações humanas que os possuem e os demais elementos dos ecossistemas que as incluem (Marques, 1995 e 2001).

A base ecológica das interações e relações humanas com o ambiente tem trazido para a ciência moderna uma grande contribuição para o conhecimento das comunidades tradicionais em seu relacionamento com a natureza, identificando e avaliando os efeitos de um sobre o outro (Almeida e Pinheiro, 2005). Neste sentido, Toledo (1992) considerou que o ponto de partida de qualquer trabalho etnoecológico deve ser a exploração das conexões entre o kosmus (sistema de crenças, visão de mundo, cosmovisão), o corpus (sistema cognitivo, repertório de informações) e a praxis (sistema de manejo, práticas).

Existe uma relação básica e tradicional entre inúmeras comunidades humanas que vivem em verdadeira simbiose com o manguezal. Em certas regiões do Nordeste, essa relação é tão intensa que Andrade (1983) afirmou existir uma "civilização do mangue", denominação dada para as populações que vivem em contato íntimo com o manguezal. Na realidade, segundo Marques (1993), constituem-se num extremo de um espectro que inclui as diversas possibilidades da inserção da politicamente plástica espécie Homo sapiens no intrincado ecossistêmico dos manguezais, onde os pescadores portam o saber e o saber-fazer relacionados com a estrutura e função do ecossistema a que estão vinculados. Trata-se, então, de um modo de vida em que atividades econômicas, sociais e culturais dependem fundamentalmente da existência do manguezal (Diegues, 1990).

Segundo Cunha (2003), o conhecimento que os pescadores possuem sobre os ecossistemas dos quais fazem parte e sobre a diversidade de espécies que ali habitam constitui um verdadeiro patrimônio que a modernidade não pode prescindir para a continuidade da vida no planeta. Na verdade, o manguezal tem importância na economia de subsistência de várias comunidades litorâneas, onde a avaliação da potencialidade do ecossistema como recurso renovável, pode servir de base para o estabelecimento da sustentabilidade das atividades pesqueiras, já que são áreas de elevada produtividade biológica assegurada pela presença de todos os elos da cadeia alimentar (Schaeffer-Novelli, 1989).

O Município de Itapissuma apresenta suas principais atividades econômicas concentradas na pesca artesanal de vários grupos de animais (Silva, 2001), com cerca de $70 \%$ da população do município dependendo direta e indiretamente desta atividade na composição da renda domiciliar e sendo considerada a maior produção de pescado do Estado de Pernambuco (Lima e Quinamo, 2000; Pedroza Júnior et al., 2002; IBAMA, 2004), além do fato de que a população obtém também diversos bens e serviços provenientes dos manguezais, tais como combustível e material de construção, que entram na composição da renda domiciliar (Schuler et al., 2000). Diante do exposto, realizou-se o presente trabalho com o objetivo de verificar a relação existente entre os pescadores artesanais de Itapisssuma e o complexo estuário-manguezal. 


\section{Material e Métodos}

\section{Área de estudo}

O Município de Itapissuma faz parte da Microrregião da Mata Setentrional de Pernambuco e integra a Região Metropolitana do Recife, situada na Latitude de 746’26"S eLongitude de 34'53'27' W, destacando-se biologicamente por possuir vastas áreas de manguezais associados ao complexo estuarino-costeiro do Canal de Santa Cruz (Medeiros e Kjerfve, 1993), considerado por Barros et al. (2000) como um dos sistemas mais importantes do litoral do Estado de Pernambuco, representando uma unidade ecológica de grande significado sócio-econômico, produtividade natural e biodiversidade. Segundo IBAMA (2004), o município contribui com $26 \%$ da produção do pescado desembarcado de Pernambuco, o que o torna o principal pólo pesqueiro no cenário pernambucano (Quinamo, 2005).

\section{Procedimentos}

Para o desenvolvimento desta pesquisa aplicouse a técnica de Candiani et al. (2004), que consiste em um levantamento de opiniões e impressões que permite investigar e descrever uma situação de uma população ou amostra pertencente à mesma podendo ser aplicada em todas as áreas do conhecimento. Neste caso, foram investigadas as impressões de 54 pescadores do sexo masculino, moradores de Itapissuma, sobre os manguezais, durante o período de novembro de 2005 a março de 2006.

Para tanto, foram elaboradas dez perguntas subjetivas, contemplando a caracterização bioecológica do manguezal, enfocando sua constituição (comunidades vegetais e animais), sua importância e benefícios para o homem e atividade de pesca, envolvendo seus métodos (incluindo tipos de iscas e materiais utilizados) e principais produtos de comercialização. Nesta pesquisa utilizou-se a diferenciação de designação entre os termos "manguezal", ambiente presente nas zonas costeiras sob a influência das marés, nas regiões intertropicais, formando uma unidade faunística e florística de muita importância ecológica e socioeconômica (Vannucci,
2002), e "mangue", espécie vegetal lenhosa típica destes ambientes (Sugiyama, 1995).

As entrevistas semi-estruturadas foram aplicadas por moradores de Itapissuma que possuíam relações familiares ou de trabalho com os pescadores da região, para estabelecer um canal de comunicação com os mesmos (rapport). Seguindo a metodologia de Costa Neto e Marques (2000) as expressões e palavras nativas foram utilizadas a fim de gerar maior confiança entre as partes. As entrevistas aconteceram durante suas atividades de pesca ou coleta dos recursos biológicos, ou logo após sua chegada ao município, sempre ocupando alguns minutos e foram documentadas de forma escrita no próprio formulário de entrevista.

As respostas foram posteriormente sistematizadas agrupando-se as expressões similares para estabelecer sua respectiva representação percentual. Foram referenciados no texto os nomes populares dos vegetais e animais citados e seus respectivos nomes científicos. Quando as perguntas subjetivas deram margem a múltiplas respostas, o percentual foi calculado a partir do quantitativo de evocações apresentadas para cada item apontado pelos pescadores, ampliando assim o universo percentual.

\section{Resultados}

As análises das respostas dos pescadores de Itapissuma revelaram um conhecimento acurado sobre a biodiversidade estuarina e sua vinculação à produção do pescado, conforme apresentado a seguir.

\section{Biodiversidade do manguezal}

No que diz respeito à biodiversidade do ecossistema manguezal os pescadores demonstraram conhecer sua vegetação característica, pois 39,3\% nomearam os quatro tipos de mangues existentes no ambiente: manguevermelho, gaiteiro, de caneta ou de casco (Rhizophora mangle); mangue-preto, siriúba ou canoé (Avicennia schaueriana); mangue-manso ou branco (Laguncularia racemosa); e mangue-de-botão (Conocarpus erectus) e $39,3 \%$ reconheceram três tipos (excluindo o mangue de botão), 3,6\% apenas dois tipos, 10,7\%, um tipo e 7,1\% desconheceram as árvores típicas. 
Em relação aos animais que se alimentam no mangue, os pescadores indicaram os seguintes: caranguejos (Ucides cordatus), aratus (Goniopsis cruentata), guaiamuns (Cardisoma guanhumi), siris (Callinectes spp.), mariscos (Anomalocardia brasiliana, Iphigenia brasiliana, Lucina pectinata, Macoma constricta, Tivela mactroides), ostras (Crassostrea rhizophorae), sururus (Mytella spp.), unhas-de-véio (Tagelus plebeius), peixes, garças (Ardea alba), socós (Nycticorax nycticorax), gaivotas (Sterna spp.), maçaricos (Calidris pusilla), siricóias (Laterallus viridis), guaxelos e guaxinins (Procyon spp.) e raposas (Cerdocyon thous).

Dos animais evocados quanto à dependência trófica existente entre eles e os mangues, destacaram-se, como os de maior representatividade, os caranguejos, apontados por $82 \%$ dos entrevistados, seguidos pelos aratus $(67,9 \%)$ e siris $(28,6 \%)$. Perguntados sobre o destino das folhas de mangue ao caírem na maré (termo utilizado localmente para designar a água estuarina), $35,7 \%$ dos entrevistados indicaram que as folhas servem de alimento para os caranguejos e aratus quando apodrecem; $28,6 \%$ assinalaram que quando as folhas apodrecem se transformam em lama e servem de alimento para os seres vivos e os fungos; $10,7 \%$ sinalizaram apenas o apodrecimento das folhas; $17,9 \%$ responderam que elas ressecam; e 7,1\% acreditam que a maré leva as folhas para o mar.

Relativamente aos benefícios do manguezal para os seres vivos, foram indicados os seguintes: alimentação para o homem (46,4\%); alimentação para os animais (42,9\%); purificação do ar e da água (10,7\%), além de outras respostas, em menor percentual, como: moradia, trabalho para as pessoas, local de desova dos animais, criação de crustáceos, extração de tinta e remédios, extração da madeira, sombra, ventos e absorção da água.

Especificamente quanto à utilidade dos mangues para o ser humano, estes foram indicados para extração de: remédios $(35,7 \%)$, tinta $(42,9 \%)$, alimentação para os animais, madeira (utilizados na confecção de barcos, armadilhas, caiçaras e habitações) $(35,7 \%)$, limpeza do ar.

\section{Atividades de pesca}

$\mathrm{Na}$ investigação sobre o pescado, $65 \%$ indicaram pescar somente peixes, incluindo uma grande diversidade: saúna (Mugil spp.), tainha-olho-preto (Mugil trichodon), curimã (Mugil lisa), manjuba (Anchoa filifera), carapeba (Diapterus rhombeus), mingula (Gobionellus oceanicus), carapicu (Eucinostomus spp.), agulha (Hyporhamphus spp.), bagre (Arius spp.), arraia (Dasyatis guttata), pacamon (Amphichthys cryptocentrus), boca-torta (Anchovia sp.), rabo-de-fogo (Anchoajanuaria), pescada (Cynoscium spp.), mero (Epinepheus itajara), camurim (Centropomus spp.) e xaréu (Caranx hippos). Em relação aos demais animais, $24 \%$ pescam só camarões (Litopenaeus schimitti e Farfantepenaeus subtilis) e os demais recursos biológicos foram apontados em pequena quantidade: siri (Callinectes spp.) (6\%), ostra (Crassostrea rhizophorae) (3\%), caranguejos (Ucides cordatus) (1\%), e sururu (Mytella charruana) (1\%).

Quanto aos métodos utilizados para a pesca, foram indicadas as redes de emalhar, de arrasto e tarrafa, para a captura de peixes e camarões; o uso de anzol e linha para a pesca de carapebas, pacamons, arraias e siris; de puçá (jereré), para agulhas; espeto, para os sururus; foice, para ostras; e coletas manuais, para captura de caranguejos. A sinalização de iscas apropriadas para pesca de determinadas espécies apontou o uso de camarão, tamarus (Alpheus spp., Callianassa spp. e Cloridopsis dubia), mingulas e carapicus para pesca de peixes maiores e o uso de vísceras de galinhas e manjubas para a captura de siris.

É oportuno mencionar que o pescado é comercializado congelado, acondicionado para venda normalmente em isopor, freezer e/ou geladeira (peixes, crustáceos e moluscos), torrado e/ou cozido (camarão) ou seco e/ou salgado (peixes).

\section{Discussão}

O etnoconhecimento dos pescadores itapissumenses sobre a biodiversidade do complexo estuário-manguezal e os mecanismos biológicos que lá se processam apresentam pontos de cruzamento com a ciência, já que é fruto de sua experiência e vivência direta com 
este ambiente. Alves e Nishida (2002) ressaltaram que comunidades tradicionais que vivem próximas aos manguezais e dependem de recursos oriundos desses ambientes apresentam um amplo conhecimento acerca dos componentes bióticos e abióticos que integram esse ecossistema. No caso das populações que exploram recursos destes ecossistemas encontram-se ainda, em muitos locais, formas tradicionais de usos do ambiente, onde as populações detêm um grande conhecimento de espécies, significando que elas têm uma boa percepção do meio em que vivem e que o princípio da sustentabilidade procura ser mantido, numa forma de simbiose com o complexo estuário-manguezal (Diegues, 1996; Adams, 2000).

Para isso, estas populações utilizam conhecimentos empíricos repassados entre as gerações e aperfeiçoados pelos membros das comunidades ao longo dos anos. Machado-Guimarães (1995) afirma que todos os grupos culturais conhecem o mundo ao seu redor, incorporando informações e observações diretas e atuais, além de saberes acumulados por antepassados e transmitidos entre as gerações por diversos mecanismos culturais. Esta autora complementa, ainda, que a atividade pesqueira artesanal requer dos pescadores um conhecimento etnoecológico que possibilita a utilização dos recursos pesqueiros que garantem a sustentabilidade desta praxis.

No que diz respeito às quatro espécies de mangues e suas denominações populares apontadas pelos pescadores, as mesmas correspondem às citadas por Sugiyama (1995), como as de maior ocorrência para o litoral brasileiro, informação esta ratificada por Coelho et al. (2004), para o Nordeste. Dahdouh-Guebas et al. (2006) obtiveram resultados semelhantes de reconhecimento das principais espécies de mangues pelos seus nomes comuns, entrevistando pescadores que atuam nas áreas estuarinas dos manguezais de Godavari, na Índia, onde $83 \%$ dos entrevistados responderam de forma boa ou muito boa, segundo a classificação dos autores, ressaltando-se a dificuldade dos entrevistados devido à biodiversidade florística daquela localidade, que compreende 13 espécies.

Em relação à fauna dependente dos mangues, os pescadores sinonimizaram os termos "mangues" (árvores) e "manguezais" (ecossistema), fato este também observado por Pereira et al. (2006) e Farrapeira et al. (2007), entrevistando estudantes de Itapissuma e do Recife, respectivamente, os quais não conseguiram atribuir diferenças entre estes dois termos. Por este motivo, a relação de animais apresentada pelos pescadores incluiu a fauna característica do manguezal (Por, 1989; Neumann-Leitão, 1995; Coelho et al., 2004) e não propriamente aquela que se alimenta dos vegetais superiores. Ainda assim, a maioria sinalizou a relação dos caranguejos com os mangues em relação a esta dependência trófica.

De acordo com Por (1989), a maior parte da produção orgânica dentro do manguezal é a proveniente das plantas superiores; neste caso, os macro-comedores, como caramujos e caranguejos, consomem o material orgânico composto por pedaços de detritos vegetais contendo a sua microflora e fauna que são processados dentro do ecossistema aquático. Já Nomura (1978) especificou que os caranguejos (Ucides cordatus) alimentam-se de vegetais superiores, algas e poríferos, enquanto que os aratus (Aratus pisonii) consomem as folhas de mangues. Estes crustáceos foram citados por uma grande quantidade de pescadores.

A percepção dos pescadores sobre a relação trófica dos caranguejos com as árvores de mangues e também sobre o destino de suas folhas no ambiente estuarino é corroborada pelas observações científicas. De acordo com Luederwaldt (1919), os caranguejos têm importância para o manguezal não apenas como um elo da cadeia alimentar ou como alimento para o homem e outros animais, mas também pelo trabalho constante de revirar a lama trazendo para a superfície a matéria orgânica que jaz em seu interior. Oliveira e Krau (1953) calcularam que os guaiamuns na Ilha de Pinheiro, no Rio de Janeiro, movimentam aproximadamente 11 toneladas de terra por semana. Legat et al. (2005) mostraram seu papel fundamental na cadeia trófica deste ambiente, promovendo a ciclagem dos nutrientes, fragmentando as folhas que caem das árvores, facilitando sua utilização por outros organismos menores. De acordo com Lira et al. (1992), parte das folhas que não são consumidas diretamente pelos caranguejos é transformada em matéria orgânica dissolvida na água, pela ação de 
fungos, bactérias e protozoários sobre as mesmas. Estes nutrientes presentes no sedimento, juntamente com bactérias e algas microscópicas, servem de alimento para outras espécies. Sua função ecológica decorre também do fato de que muitas espécies escavam suas tocas, onde se abrigam e armazenam alimento, como um verdadeiro labirinto subterrâneo (Olmos e Silva, 2003), induzindo, desta forma, a um fluxo de matéria orgânica da superfície para o subterrâneo e deste transportando matéria inorgânica para a superfície, promovendo aeração, drenagem dos sedimentos e enriquecimento do solo, tornando-os menos compactados e facilitando a penetração e desenvolvimento das raízes das plantas nele existentes (Alves e Nishida, 2004).

Quanto aos benefícios do manguezal para os seres vivos, os pescadores possuem uma concepção acurada, reconhecendo a importância deste ecossistema para suas vidas, pela pluralidade de respostas apresentadas. Segundo Diegues (1996), como as sociedades tradicionais conhecem profundamente a natureza elas se caracterizam pela dependência e até simbiose com a natureza, os ciclos e os recursos naturais renováveis a partir dos quais se constrói um modo de vida. As respostas dos pescadores encontraram, portanto, similaridades com as observações de Alves e Nishida (2002), que ressaltaram que as comunidades tradicionais que vivem próximas aos manguezais e dependem de recursos oriundos desses ambientes apresentam um amplo conhecimento acerca dos componentes bióticos e abióticos que integram esses ecossistemas.

De um modo geral, relativamente ao uso das plantas pelo homem, as respostas obtidas resgataram a afirmação de Marques (1993), que sinalizou ser nos manguezais que muitas populações obtêm produtos de pesca, carvão vegetal e produtos derivados como taninos e plantas medicinais, em um processo tradicional. Um outro destaque fornecido pela população entrevistada foi o uso dos mangues para a extração de remédios, fato este também observado por Premanathan et al. (1999), que afirmaram que o uso de plantas medicinais é uma prática comum em regiões de manguezais mundialmente, sendo considerada uma interferência modesta, de pequena expressividade para o ambiente. $\mathrm{O}$ conhecimento sobre plantas medicinais simboliza, não raro, o único recurso terapêutico de muitas comunidades. Observa-se que localidades onde o acesso à saúde formal é limitado, o uso deste recurso como forma de atenção básica à saúde é intensificado, pois oferece uma terapêutica de custo reduzido em relação à proporcionada por medicamentos alopáticos (Victor e Andrade, 1991; Jouad et al., 2001).

Quanto à pesca, as espécies de peixes citadas pelos pescadores de Itapissuma foram, em grande parte, também relacionadas por El-Deir (1999), no estudo etnoecológico da comunidade de Vila Velha, próxima à Barra Orange do Canal de Santa Cruz e por Vasconcelos Filho e Oliveira (1999) para os estuários de Pernambuco. Esta mesma ictiofauna foi coincidente ainda com a observada por Maia e Cavalcante (2005) em 17 estuários do Ceará e também por Mourão e Nordi (2002 e 2003), para o estuário de Mamanguape, Paraíba. A denominação popular dada aos peixes em todas estas localidades é semelhante e tal fato pode ser justificado pelas raízes culturais comuns às áreas litorâneas do Nordeste do Brasil e da interculturalidade dos pescadores que transitam e comercializam seus produtos nesta região.

No que diz respeito à ausência de citações aos mariscos e à baixa quantidade de menções dos demais moluscos e crustáceos pelos entrevistados, estas se devem à escolha prévia da pesquisa em entrevistar apenas pescadores do sexo masculino. Lima e Quinamo (2000) e Pedroza Júnior et al. (2002) observaram para a área do Canal de Santa Cruz uma divisão de tarefas ligadas ao gênero: a pesca com barcos e redes é uma tradição dos homens $(100 \%$ dos entrevistados deste trabalho) e a mariscagem e captura de caranguejos, siris, sururus e ostras, atribuição das mulheres e crianças.

A escolha das iscas utilizadas pelos pescadores de Itapissuma demonstrou um relativo conhecimento destes sobre as relações tróficas que ocorrem no ambiente estuarino. Algo semelhante ao que foi observado em duas localidades na Bahia. Costa Neto et al. (2002) constataram que os pescadores de Barra conhecem os hábitos alimentares das etnoespécies, classificando os peixes de acordo com os itens alimentares mais mencionados em duas categorias gerais: peixes especialistas, para os quais foram citados uma ou duas 
categorias de alimentos, e peixes generalistas, para os quais foram mencionados diferentes itens alimentares. Souto e Marques (2006) relataram as observações ecológicas dos pescadores de Acupe sobre os hábitos alimentares dos siris que são comparáveis às conhecidas pela ciência.

Em relação aos principais equipamentos de pesca e técnicas usados pelos pescadores de Itapissuma em sua prática cotidiana, estes correspondem aos utilizados especificamente por pescadores do sexo masculino, conforme citado por vários autores que trabalharam a questão etnobiológica e sócio-ambiental desse grupo pesquisado (El-Deir, 1999; Silva, 2001; Pedroza Júnior et al., 2002; Albuquerque e Albuquerque, 2005).

A maior parte do pescado é comercializada no mercado de peixe que o município possui. Segundo Pedroza Júnior et al. (2002), o produto da pesca pode ainda ser vendido do pescador diretamente para bares e restaurantes e para consumidores que encomendam os animais. Os métodos indicados para conservação, estocagem e acondicionamento do pescado para a venda são similares aos observados por Silva (2001), na mesma localidade.

A pesquisa evidenciou que os pescadores da comunidade tradicional do Canal de Santa Cruz possuem um conhecimento acurado do complexo estuáriomanguezal, especificamente de sua biodiversidade e utilidade, o que permitiu a sua produção e reprodução social no tempo, bem como a manutenção e conservação dos próprios recursos naturais de que dependem para sobreviver. Este conhecimento reflete, portanto, uma bagagem cultural onde a arte da pesca é transmitida oralmente da geração ascendente aos descendentes, advindas por meio de observações e aprendizagem prática.

\section{Referências}

Adams, C. 2000. As populações caiçaras e o mito do bom selvagem: a necessidade de uma nova abordagem interdisciplinar: Revista de Antropologia, 43 (1): 146-182.

Albuquerque, C. A.; Albuquerque, U. P. 2005. Local perceptions towards biological conservation in the community of Vila Velha, Pernambuco, Brazil. Interciencia, 30 (8): 460-465.

Almeida, I. C. S.; Pinheiro, C. U. B. 2005. Uso do conhecimento tradicional na identificação de indicadores de mudanças ecológicas nos ecossistemas aquáticos da região lacustre de Penalva, Área de proteção Ambiental da baixada Maranhense - I. Peixes. In: Alves, A. G.; Lucena, R. F. P. \& Albuquerque, U. P. (Eds). Atualidades em etnobiologia e etnoecologia. NUPEEA/Sociedade Brasileira de Etnobiologia e Etnoecologia, Recife, Brasil, p.61-80.

Alves, R. R. N.; Nishida, A. K. 2002. A ecdise do caranguejo-uçá, Ucides cordatus (Crustacea, Decapoda, Brachyura) na visão dos caranguejeiros. Interciencia, 27 (3): 110- 117.

Alves, R. R. N.; Nishida, A. K. 2004. Population structure of the mangrove crab Ucides cordatus (Crustacea: Decapoda; Brachyura) in the estuary of the Mamanguape river, Northeast Brazil. Tropical Oceanography, 32 (1): 23-37.

Andrade, G. O. 1983. Fenômeno da superurbanização do Nordeste. In: Jatobá, L. (Org.). Estudos nordestinos sobre crescimento urbano. Fundação Joaquim Nabuco, Recife, Brasil, p.21-44.

Barros, H. M.; Leça, E. E.; Paranaguá, M. N. 2000. Gestão comunitária de recursos naturais: Ampliando competências locais para o tratamento sustentável da questão litorânea no Nordeste. Trabalhos completos do MANGROVE 2000: Sustentabilidade de Estuários e Manguezais: Desafios e Perspectivas, Recife, Brasil, p.10.

Begossi, A.; Figueiredo, J. L. 1995. Ethnoichthyology of southern coastal fishermen: Cases from Buzios Island and Sepetiba Bay (Brazil). Bulletin of Marine Science, 5 (2): 710-717.

Candiani, G.; Lage, M.; Vita, S.; Souza, W.; Filho, W. 2004. Educação ambiental: Percepção e práticas sobre meio ambiente de estudantes do ensino fundamental e médio. Revista Eletrônica do Mestrado em Educação Ambiental, 12: 74-89. Disponível em $<$ http://www.fisica.furg.br/mea/remea/vol12/art07.pdf $>$. Acesso em 21 de novembro de 2006.

Coelho, P. A.; Batista-Leite, L. M. A.; Santos, M. A. C.; Torres, M. F. A. 2004. O manguezal. In: Eskinazi-Leça, E.; Neumann-Leitão, S. \& Costa, M. F. (eds). Oceanografia: Um cenário tropical. Bagaço, Recife, Brasil, p.641-688.

Costa Neto, E. M.; Dias, C. V.; Melo, M. N. 2002. O conhecimento ictiológico tradicional dos pescadores da cidade de Barra, região do médio São Francisco, Estado da Bahia, Brasil. Acta Scientiarum Biological Science, 24 (2): 561-572.

Costa Neto, E. M.; Marques, J. G. W. 2000. A etnotaxonomia de recursos ictiofaunísticos pelos pescadores da comunidade de Siribinha, Norte do Estado da Bahia, Brasil. Biociências, 8 (2): 61-76.

Cunha, L. H. O. 2003. Saberes patrimoniais pesqueiros. Desenvolvimento e Meio Ambiente, 7: 69-76. Disponível em <http://calvados.c3sl.ufpr.br/ojs2/ index.php/made/article/view/3044/2435>. Acesso em 12 de dezembro de 2006.

Dahdouh-Guebas, F.; Collin, S.; Lo Seen, D.; Rönnbäck, P.; Depommier, D.; Ravishankar, T.; Koedam, N. 2006. Analysing ethnobotanical and fishery-related importance of mangroves of the East-Godavari Delta (Andhra Pradesh, India) for conservation and management purposes. Journal of Ethnobiology and Ethnomedicine, 2: 24-46.

Diegues, A. C. S. 1990. Comunidades litorâneas e os manguezais no Brasil. PPCAUB - Série Trabalhos e Estudos, São Paulo, Brasil, 22pp.

Diegues, A. C. S. 1996. Ecologia humana e planejamento em áreas costeiras. Núcleo de Apoio à Pesquisa sobre Populações Hu- 
manas e Áreas Úmidas Brasileiras - NUPAUB - USP, São Paulo, Brasil, 191pp.

El-Deir, S. G. 1999. Gestão ambiental. I - Percepção ambiental e caracterização sócio-econômica e cultural da comunidade de Vila Velha, Itamaracá - PE (Brasil). Trabalhos Oceanográficos da Universidade Federal de Pernambuco, 27 (1): 175-185.

Farrapeira, C. M. R.; Silva, K. M. E.; Lima, A. O. 2007. Percepção e concepção do manguezal vinculados ao ensino da Biologia em uma escola de Recife - PE. Educação Ambiental em Ação, 19: 1-9. Disponível em <http://www.revistaea.arvore.com.br/ artigo.php?idartigo $=449 \&$ class $=20>$. Acesso em 27 de setembro de 2007.

IBAMA. 2004. Boletim estatístico da pesca marítima e estuarina do Nordeste do Brasil - 2003. Centro de Pesquisa e Gestão de Recursos Pesqueiros do Litoral Nordeste - CEPENE/ IBAMA, Tamandaré, Brasil, 191pp.

Jouad, H.; Haloui, M.; Rhiouani, H.; El Hilaly, J.; Eddouks, M. 2001. Ethnobotanical survey of medicinal plants used for the treatment of diabetes, cardiac and renal diseases in the North centre region of Morocco (Fez-Boulemane). Journal of Ethnopharmacology, 77 (2-3): 175-182.

Legat, J. F. A.; Raseira, M. B.; Marino, M. V.; Mota, R. I.; Moura Neto, D.; Daniel, R. 2005. Avaliação da captura do caranguejo-uçá, Ucides cordatus, através do método tradicional e com a utilização de armadilhas tubulares. Resumos do II Congresso Brasileiro de Oceanografia, Vitória, Brasil, p.3.

Lima, T.; Quinamo, T. 2000. Características sócio-econômicas. In: Barros, H. M.; Eskinazi-Leça, E.; Macedo, S. J. \& Lima, T. (Eds). Gerenciamento participativo de estuários e manguezais. Ed. Universitária da UFPE, Recife, Brasil, p.181-225.

Lira, A.; Sá, H. P.; Amador, J.; Cavalcanti, R. 1992. Manguezais, importância de sua preservação. Aspectos da degradação dos manguezais de Pernambuco. Escola Recanto, Recife, Brasil, $87 \mathrm{pp}$.

Luederwaldt, H. 1919. Os manguezaes de Santos. Revista do Museu Paulista, 1: 309-408.

Machado-Guimarães, E. M. 1995. A pesca tradicional em Maricá (RJ) sob uma perspectiva da ecologia cultural. Tese de Doutorado, Universidade Federal de São Carlos, Brasil, 109pp.

Maia, L. D.; Cavalcante, M. D. (Coords). 2005. Ictiofauna dos estuários do estado do Ceará. Superintendência Estadual do Meio Ambiente - SEMACE, Fortaleza, Brasil, 200pp. Disponível em $<$ http://www.semace.ce.gov.br/programas/zee/Produtos/ RelatórioICTIOFAUNA.pdf $>$. Acesso em 24 de janeiro de 2007.

Marques, J. G. 1995. Pescando pescadores: Etnoecologia abrangente no Baixo São Francisco. Núcleo de Apoio à Pesquisa sobre Populações Humanas e Áreas Úmidas Brasileiras NUPAUB-USP, São Paulo, Brasil, 285pp.

Marques, J. G. 2001. Pescando pescadores: Ciência e etnociência em uma perspectiva ecológica. Núcleo de Apoio à Pesquisa sobre Populações Humanas e Áreas Úmidas Brasileiras - NUPAUB USP, São Paulo, Brasil, 258pp.

Marques, J. G. W. 1993. Etnoecologia, educação ambiental e superação da pobreza em áreas de manguezais. Anais do I Encontro Nacional de Educação Ambiental em Áreas de Manguezais, Maragogipe, Brasil, p.29-35.
Medeiros, C.; Kjerfve, B. 1993. Hydrology of a tropical estuarine system: Itamaracá, Brazil. Estuarine, Coastal and Shelf Science, 36: 495-515.

Mourão, J. S.; Nordi, N. 2002. Comparações entre as taxonomias folk e científica para peixes do estuário do Rio Mamanguape, Paraíba-Brasil. Interciencia, 27 (12): 664-668. Disponível em <http:// www.interciencia.org/v27_12/mourao.pdf $>$. Acesso em 24 de janeiro de 2007.

Mourão, J. S.; Nordi, N. 2003. Etnoictiologia de pescadores artesanais do estuário do Rio Mamanguape, Paraíba-Brasil. Boletim do Instituto de Pesca, 29 (1): 9-17.

Neumann-Leitão, S. 1995. A fauna do manguezal. In: SchaefferNovelli, Y. (Ed.). Manguezal: Ecossistema entre a Terra e o mar. Caribbean Ecological Research, São Paulo, Brasil, p.23-27.

Nishida, A. K. 2005. Etnoecologia de manguezais. In: Alves, A. G.; Lucena, R. F. P. \& Albuquerque, U. P. (Eds). Atualidades em etnobiologia e etnoecologia. NUPEEA/Sociedade Brasileira de Etnobiologia e Etnoecologia, Recife, Brasil, p.183-194.

Nomura, H. 1978. Criação de moluscos e crustáceos. Nobel, São Paulo, Brasil, 102pp.

Oliveira, L. P. H.; Krau, L. 1953. Levantamento biogeográfico da Baía de Guanabara. II - Crescimento do manguezal na Ilha do Pinheiro. Memórias do Instituto Oswaldo Cruz, 53: 503-524.

Olmos, F.; Silva, R. S. 2003. Guará: Ambiente, flora e fauna dos manguezais de Santos- Cubatão Brasil. Empresa das Artes, São Paulo, Brasil, 216pp.

Pedroza Júnior, H. S.; Soares, M. G.; Melo-Júnior, M.; Barros, H. M.; Soares, A. 2002. Aspectos etnobiológicos da pesca e comercialização de moluscos e crustáceos do Canal de Santa Cruz, Itapissuma - PE. Anais do I Congresso Brasileiro de Extensão Universitária, João Pessoa, Brasil, p.1-7. Disponível em $<\mathrm{http}$ ://www.prac. ufpb.br/anais/anais/meioambiente/moluscos.pdf $>$. Acesso em 21 de dezembro de 2005.

Pereira, E. M.; Farrapeira, C. M. R.; Pinto, S. L. 2006. Percepção e educação ambiental sobre manguezais em escolas públicas da Região Metropolitana do Recife. Revista Eletrônica do Mestrado em Educação Ambiental, 17: 244-261. Disponível em <http:// www.remea.furg.br/edicoes/vol17/art37v17a15.PDF>. Acesso em 11 de fevereiro de 2007.

Por, F. D. 1989. Guia ilustrado do manguezal brasileiro. Instituto de Biociências da USP, São Paulo, Brasil, 34pp.

Posey, D.A. 1997. Etnobiologia: Teoria e prática. In: Ribeiro, B. G. (Ed.). Suma etnológica brasileira: 1. Etnobiologia. Ed. Universitária UFPA, Belém, Brasil, p.5-15.

Premanathan, M.; Kathiresan, K.; Nakashima, H. 1999. Mangrove halophytes: A source of antiviral substances. South Pacific Study, 19 (1-2): 49-57.

Quinamo, T. S. 2005. Relevância socioeconômica e aspectos ambientais da pesca tradicional realizada a partir de Itapissuma, no Canal de Santa Cruz - PE. Trabalhos Científicos do IV EREAAM - Encontro Regional de Educação Ambiental em Áreas de Manguezais - Nordeste II, Recife, Brasil, p.63-65.

Schaeffer-Novelli, Y. 1989. Perfil dos ecossistemas litorâneos brasileiros, com especial ênfase sobre o ecossistema manguezal. Publicação Especial do Instituto Oceanográfico, 7: 1-16. 
Schuler, C. A. B.; Andrade, V. C.; Santos, D. S. 2000. O manguezal: Composição e estrutura. In: Barros, H. M.; Eskinazi-Leça, E.; Macedo, S. J. \& Lima, T. (Eds). Gerenciamento participativo de estuários e manguezais. Ed. Universitária da UFPE, Recife, Brasil, p.27-38.

Silva, A. J. 2001. Estudo sócio-ambiental na margem urbana do Canal de Santa Cruz -Itapissuma, Pernambuco - Brasil. Dissertação de Mestrado, Universidade Federal da Paraíba, Brasil, 170pp.

Souto, F. J. B.; Marques, J. G. W. 2006. “O siri labuta muito!” Uma abordagem etnoecológica abrangente da pesca de um conjunto de crustáceos no manguezal de Acupe, Santo Amaro, Bahia, Brasil. Sitientibus - Série Ciências Biológicas (Etnobiologia), 6: 106-119.
Sugiyama, M. 1995. A flora do manguezal. In: Schaeffer-Novelli, Y. (Ed.). Manguezal: Ecossistema entre a Terra e o mar. Caribbean Ecological Research, São Paulo, Brasil, p.17-21.

Toledo, V. M. 1992. What is ethnoecology? Origins, scope and implications of a rising discipline. Etnoecológica, 1 (1): 5-21.

Vannucci, M. 2002. Os manguezais e nós: Uma síntese de percepções. São Paulo: EDUSP, 244p.

Vasconcelos Filho, A. L.; Oliveira, A. M. E. 1999. Composição e ecologia da ictiofauna do Canal de Santa Cruz (Itamaracá - PE, Brasil). Trabalhos Oceanográficos da Universidade Federal de Pernambuco, 27 (1): 101-113.

Victor, P.; Andrade, L. H. C. 1991. Flora medicinal: Estudo comparativo entre dois municípios de Pernambuco. Biologica Brasilica, 3 (2): 179-200. 